Supplement of Saf. Nucl. Waste Disposal, 1, 11-12, 2021

https://doi.org/10.5194/sand-1-11-2021-supplement

(c) Author(s) 2021. CC BY 4.0 License.

(c) (i)

Supplement of

\title{
Developing a radiation field-based monitoring system for the transport and storage cask inventory during extended interim storage
}

Mira Stephan et al.

Correspondence to: Mira Stephan (mira.stephan@hszg.de) and Michael Wagner (michael.wagner3@tu-dresden.de)

The copyright of individual parts of the supplement might differ from the article licence. 




Developing a radiation-field-based monitoring system for the transport and storage cask inventory during extended interim storage

Author: M. Stephan ${ }^{1}$, S. Reinicke ${ }^{1}$, A. Kratzsch ${ }^{1}$, M. Wagner ${ }^{2}$, S. Kobelt' ${ }^{2}$, U. Hampel ${ }^{2,3}$

IInstitute for Process Technology, Process Automation and Measurement Technology, Hochschule Zittau/Göriltz - University of Applied Sciences, Zittau, 02763, Germany

${ }^{2}$ Chair of Imaging Techniques in Energy and Process Engineering, Institute of Power Engineering,
${ }^{3}$ Institute of Fluid dynamics, Helmholtz-zentrum Dresden-Rossendorf, Dresden, 01328 , Germany

Corresponding Author: mira.stephan@hszg.de, Michael.Wagner3@tu-dresden.de

Situation in Germany

Expiring licence:

Transport and storage cask licence limited to 40 years $\rightarrow$ begin to expire in the 2030s /2, S.90/ No operational long-term storage facility: Long-term storage facility yet to be found $\rightarrow$ not available before $2050 / 3$, S.42/

Prolonged interim storage

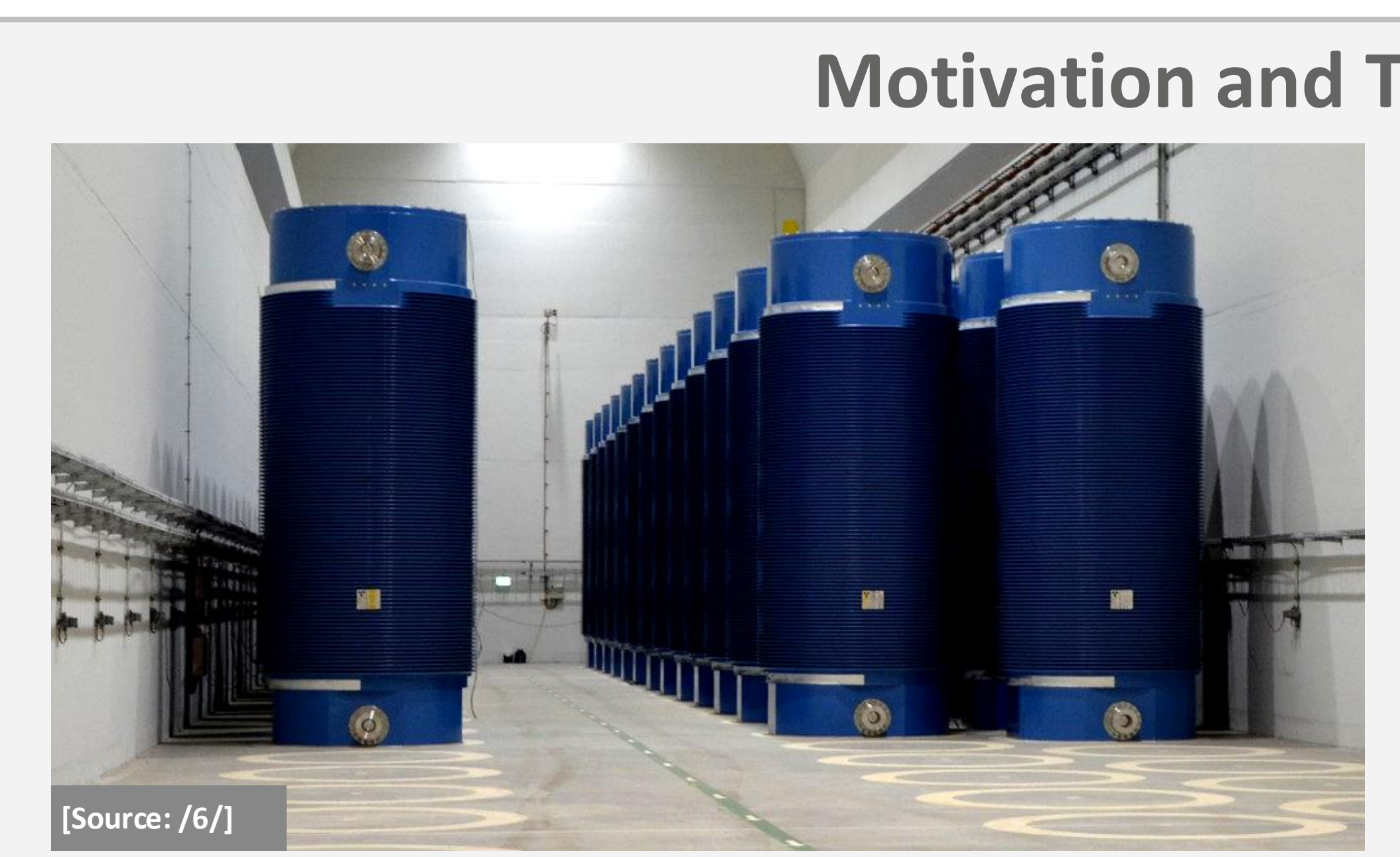

Investigation of non-invasive cask monitoring concepts

Radiation-field-based concepts promising /4, 5/:

$\rightarrow$ Investigation of gamma- and neutron-fields for major inventory changes $\rightarrow$ Construction of a partially automated gamma and neutron detector $\rightarrow$ Investigation of cosmic muon scattering for major inventory changes

$\rightarrow$ Implementation of a suitable procedure for the inverse problem in muon imaging

$\rightarrow$ Construction of a suitable muon detector

Support safety during prolonged interim storage and elongation of approval

\section{Gamma- and Neutron-Fields: Simulation}

Radiation-field outside CASTOR ${ }^{\circledR}$ V/19-cask at the cylinder surface (simulated with MCNP6.2) - Spent nuclear fuel: medium burn-up of $56.79 \mathrm{GWd} / \mathrm{tHM}$ and 5 years cooling time

- Fuel distribution changes: $\rightarrow$ axial redistribution at different fuel assembly positions $\rightarrow$ assumed vertical subsidation is $9 \mathrm{~cm}$

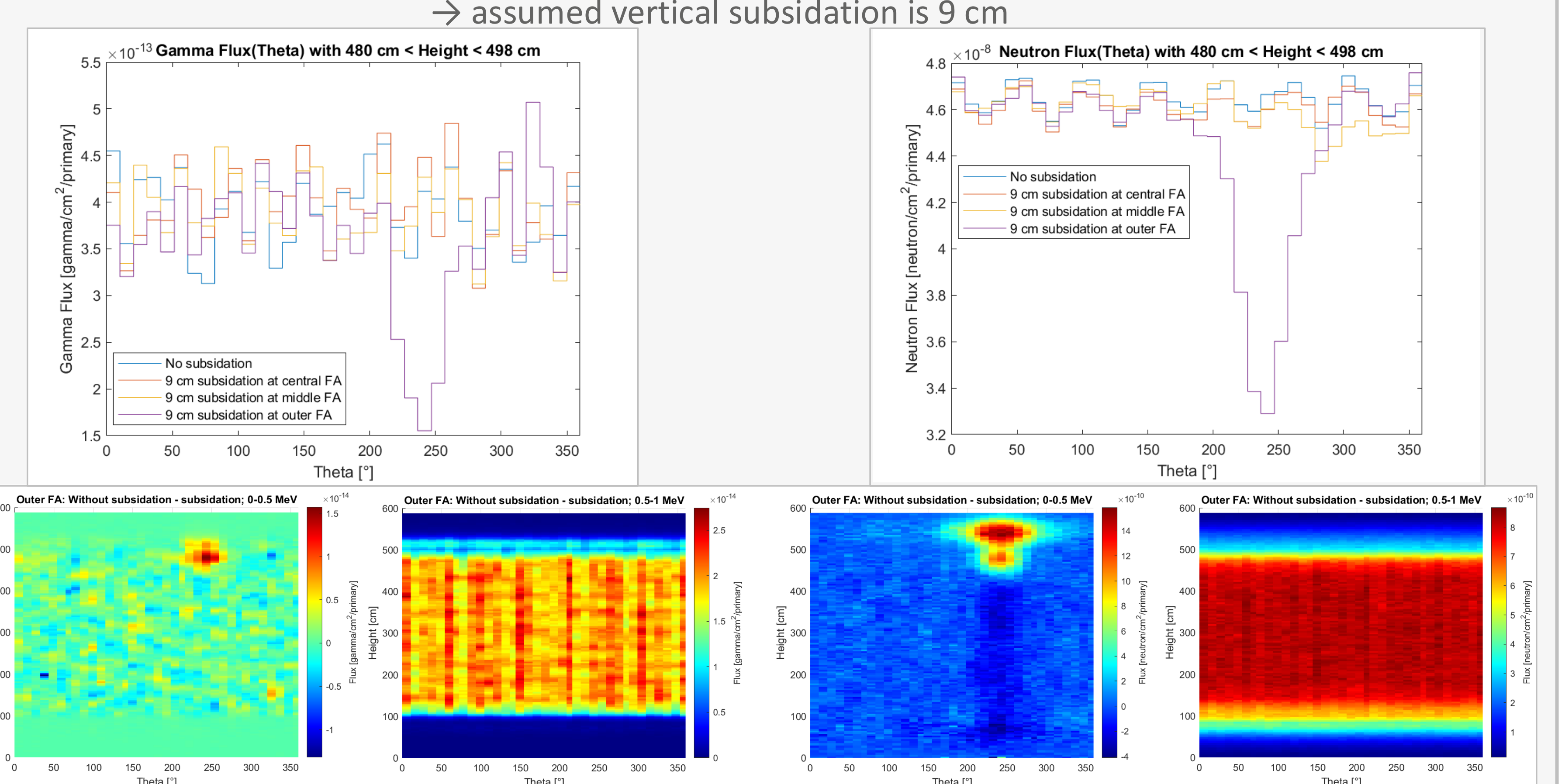

- Gamma-field shows only changes
at outer fuel assembly
- Subsiduation more recognizable at
lower energies

Gamma- and Neutron-Fields: Measurment System

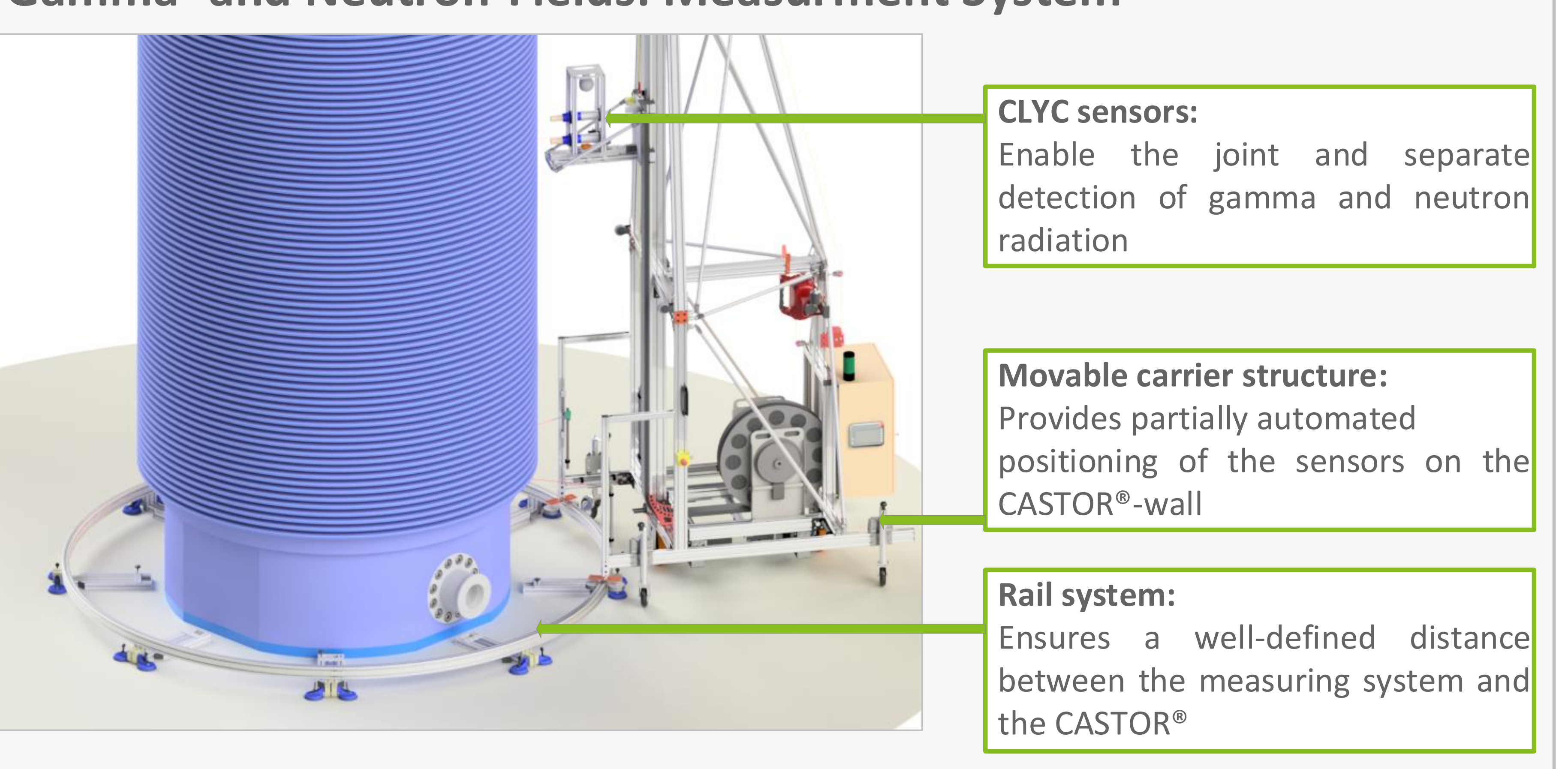

\section{Cosmic Muons: Volume Reconstruction}

Maximum likelihood estimation:

- Discretization of the object

- Assume path through the object (PoCA or more likely path)

- Calculate the muon path length for every muon and voxel "system matrix"

- Solve the linear equation system with measured data $f(L, D) \lambda=D$

Full container simulation:

- Simulation of cosmic muons with G4beamline

- Measurement time $\approx 12 \mathrm{~h}\left(3.3^{*} 10^{6}\right.$ events)

- MLEM-reconstruction with region clustering

"regularization", voxel size $\approx 6 \times 6 \times 4.5 \mathrm{~cm}^{3}$

Higher image quality

Knowledge about the object included

$\downarrow$ Higher computational effort

$\downarrow$ The usual numerical problems
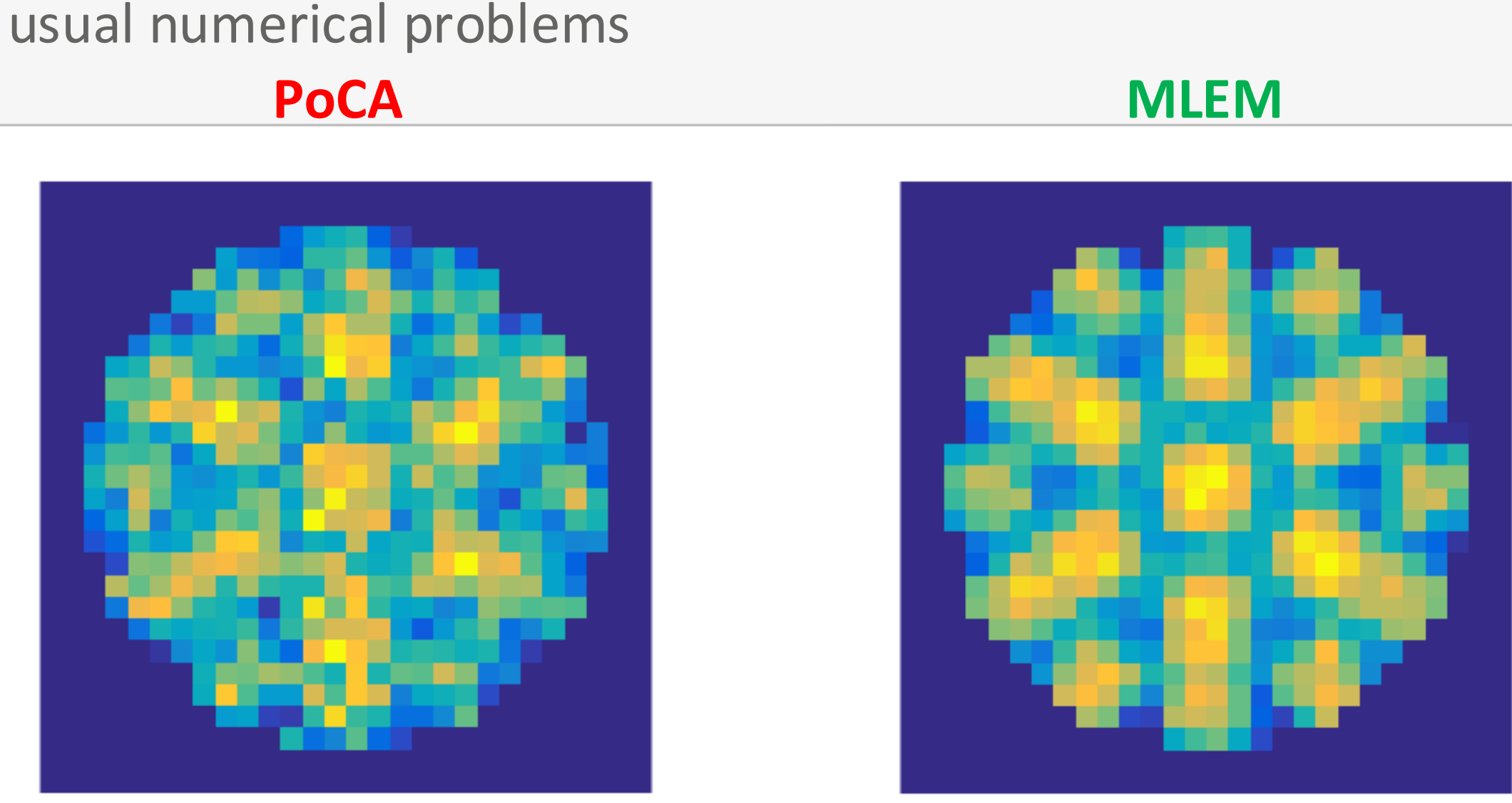

What is a cosmic muon?

Mean momentum $\approx 3 \mathrm{GeV} / \mathrm{c}$

$\rightarrow$ highly penetrating particle

Flux $\approx 1 \mathrm{~cm}^{-2} \mathrm{~min}^{-1}$

Mass $\approx 207 \mathrm{~m}$

- Interaction with matter

$\rightarrow$ energy loss and multiple

coulomb scattering

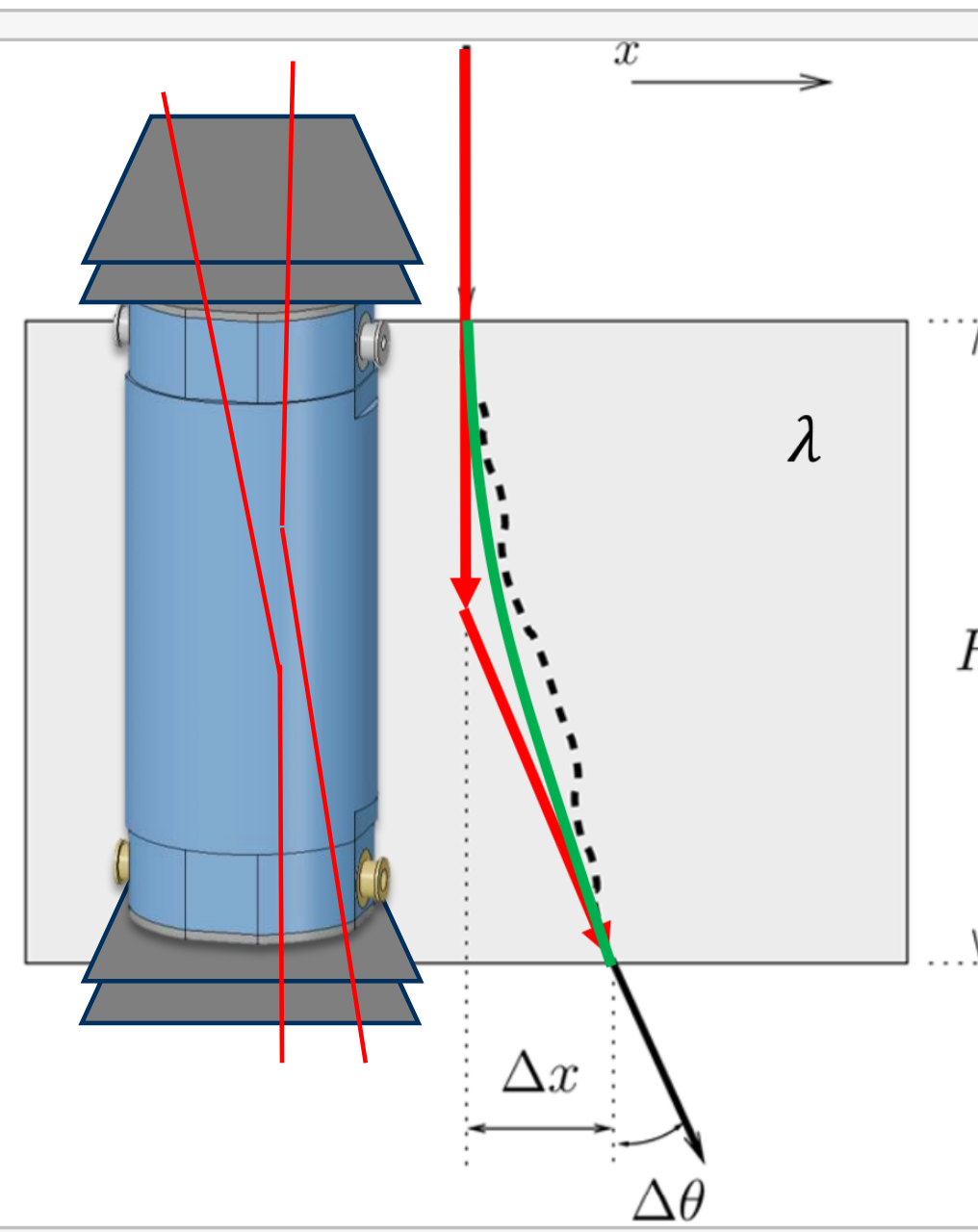

Simulation with fuel relocation

$.9 \mathrm{~cm}$ vertical subsiduation $\rightarrow$ Vertical scattering density profile of central fuel assembly (normalized)

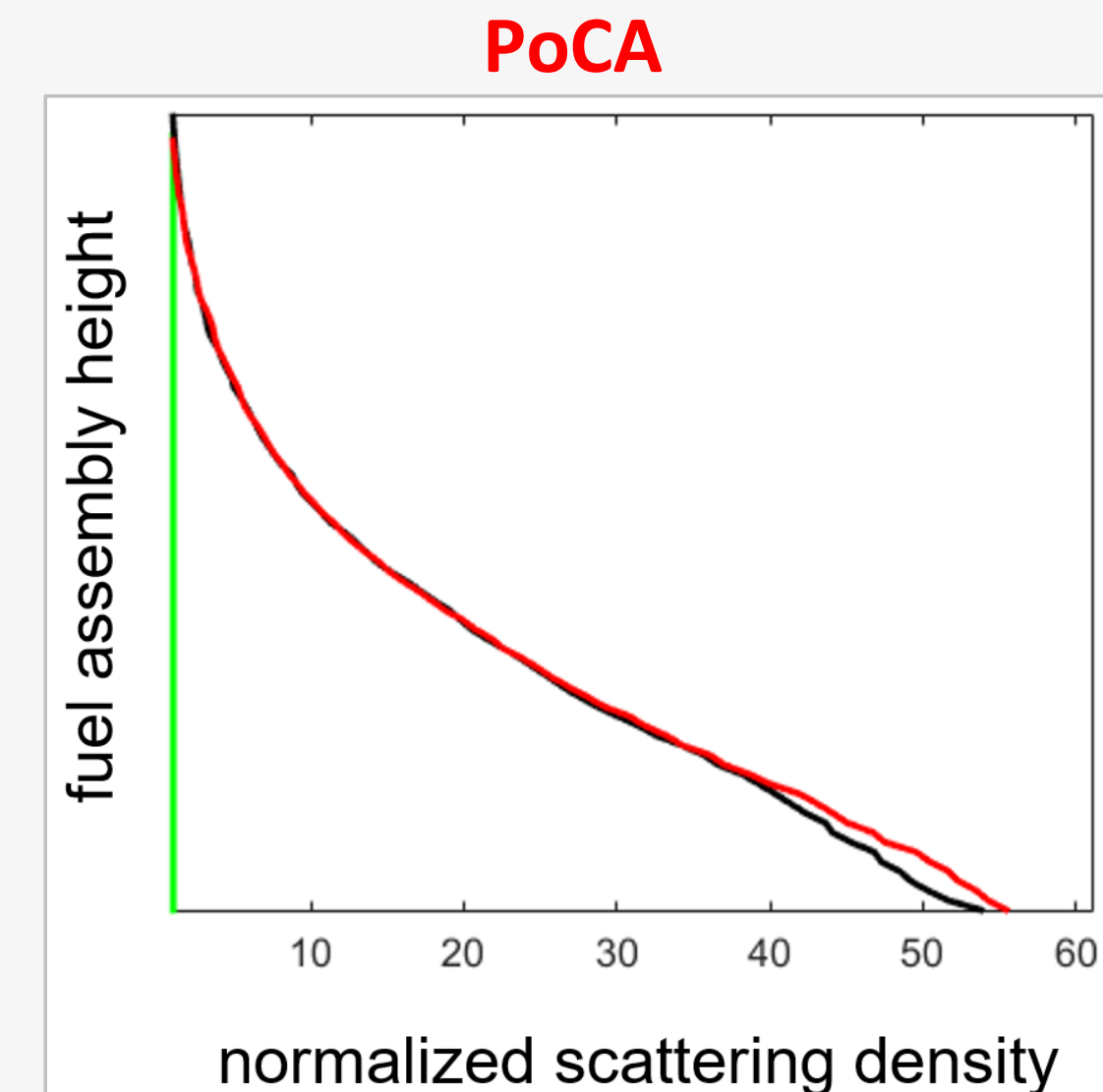

MLEM

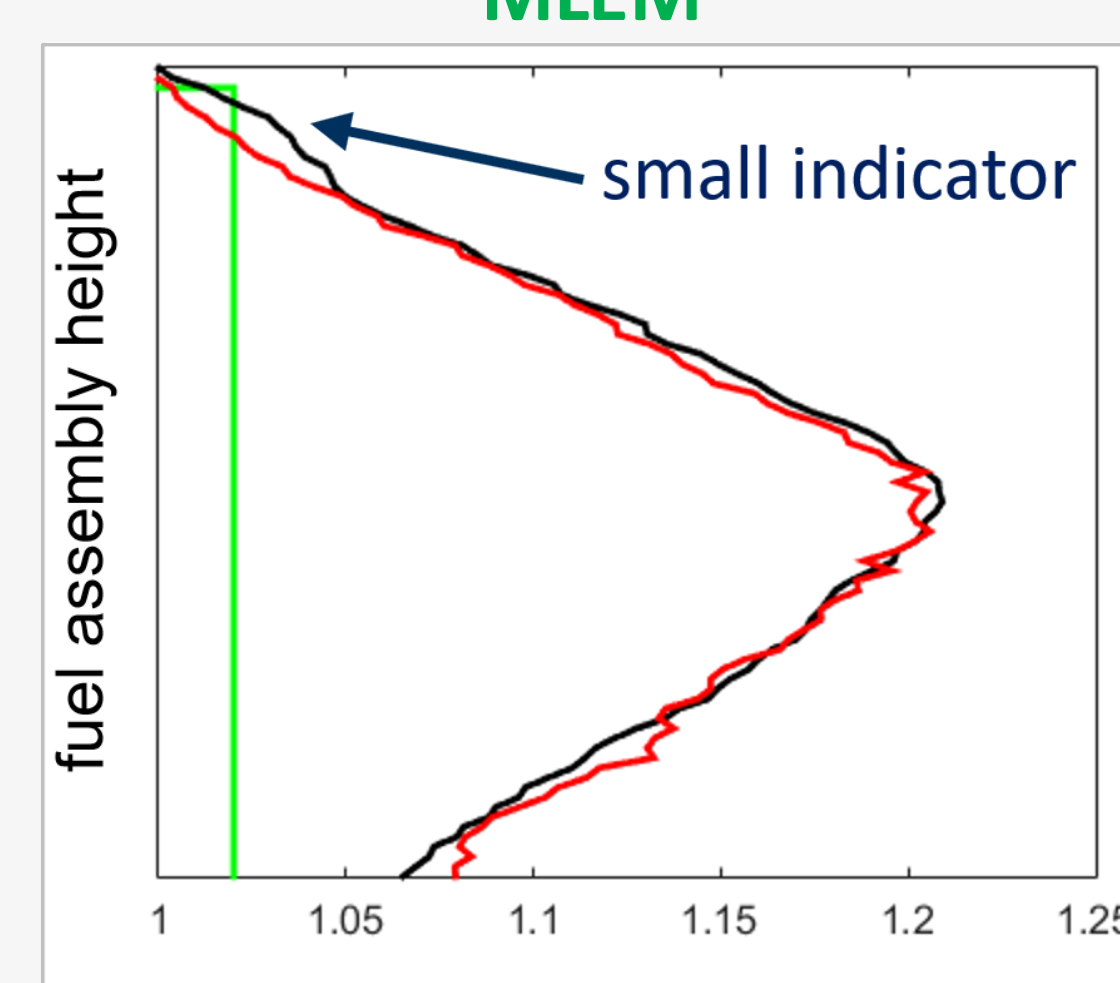

normalized scattering density

Algebraic volume reconstruction identifies fuel relocation

- Further development necessary for direct localization without reference

$\rightarrow$ Construction of a muon detector and measurement at large scale geometri (Drift chambers preferred at the moment)

\section{References}

/1/ Gesellschaft für Nuklear-Service: Behälterbeladung, URL: https://www.gns.de/language=de/21562/behaelterbeladung [Abrufdatum: 12.10.2021]

12/ Kommission Lagerung hoch radioaktiver Abfälle: Verantwortung für die Zukunft. Ein faires und transparentes Verfahren für die Auswahl eines Endlagers, Deutscher Bundestag 18. Wahlperiode,

Drucksache 18/9100, 2016

/3/ Bundesamt für die Sicherheit der nuklearen Entsorgung: Unsere Forschungsagenda, 2019

/4/ R. Rachamin and U. Hampel: Feasibility assessment of using external neutron and gamma radiation measurements for monitoring the state of the assemblies in dry storage casks,

Annals of Nuclear Energy 135, 2020

/5/ M. Wagner, R. Rachamin, S. Schmidt, D. Fiß, S. Reinicke, A. Kratzsch and U. Hampel: Simulation-based investigation of suitability of thermography and muon flux measurements for non-invasive monitoring of transport and storage containers for spent fuel, 49th Annual Meeting on Nuclear Technology (AMNT 2018), 29.-30.05.2018, Berlin, Deutschland

6/ Ministerium für Umwelt, Klima und Energiewirtschaft Baden-Württemberg: Zwischenlagerung der Abfälle. Zwischenlager Neckarwestheim, URL: https://um.baden-wuerttemberg.de/de/umwelt-natur/

kernenergie-und-strahlenschutz/entsorgung/zwischenlagerung/zwischenlager-neckarwestheim/ [Abrufdatum: 18.10.2021]

\section{Acknowledgments}

The investigations are funded by the German Federal Ministry of Economic Affairs and Energy on the basis of a decision by the German Bundestag. Grant identification number: 1501606A and 1501606B

The authors gratefully acknowledge the GWK support for funding this project by providing computing time through the Center for Information Services and HPC (ZIH) at TU Dresden

丹ipm.hszg.de

F
Institut für Prozesstechnik, Prozessautomatisierung und Messtechnik

Prof. Dr.-Ing. Alexander Kratzsch

$+49(0) 35836124282$

a.kratzsch@hszg.de 